\title{
Proximal colon cancers and the serrated pathway: a systematic analysis of precursor histology and BRAF mutation status
}

\author{
Deepa T Patil ${ }^{1}$, Bonnie L Shadrach ${ }^{1}$, Lisa A Rybicki ${ }^{2}$, Brandie H Leach ${ }^{3}$ and Rish K Pai ${ }^{1}$ \\ ${ }^{1}$ Department of Anatomic Pathology, Cleveland Clinic, Cleveland, OH, USA; ${ }^{2}$ Department of Quantitative \\ Health Sciences, Cleveland Clinic, Cleveland, OH, USA and ${ }^{3}$ Department of Genomic Medicine Institute, \\ Cleveland Clinic, Cleveland, OH, USA
}

\begin{abstract}
Although the serrated neoplasia pathway is thought to give rise to the majority of sporadic microsatellite instability-high (MSI-H) colon cancer, the exact proportion of these tumors that arise from serrated precursors has not been fully studied. Tubular and tubulovillous adenomas with features of the serrated neoplasia pathway have been described, and unlike sessile serrated adenomas, these lesions lack BRAF mutations. The contribution of these adenomas to sporadic MSI-H colon cancer is unclear. To this end, we conducted an analysis of right-sided sporadic MSI-H and microsatellite stable (MSS) colon cancer, with emphasis on precursor lesions. Overall $25 \%$ (19/75) of MSI-H colon cancer had a precursor, of which only 4 were recognized histologically as arising from a sessile serrated adenoma, and the remaining were best classified as adenomas. Of the 31 (of 89 ) MSS colon cancers with a precursor, only 1 was a sessile serrated adenoma $(P=0.06)$. Histological analysis of the precursor adenomas to sporadic MSI-H colon cancer demonstrated a high frequency of crypt serrations compared with MSS colon cancer (93 vs $36 \%, P<0.001)$. BRAF mutations were found in $57 / 75(76 \%)$ sporadic MSI-H and 10/89 (11\%) MSS colon cancers $(P<0.001)$. Molecular analysis demonstrated BRAF mutations in 11/12 adenoma and $3 / 3$ sessile serrated adenoma precursors adjacent to BRAF-mutated MSI-H colon cancer. Similarly, all 4 precursors to BRAF-mutated MSS colon cancer were also BRAF mutated. The presence of BRAF mutations in these adenomatous precursors suggests that they represent sessile serrated adenomas with complete cytologic dysplasia. Finally, patients with sporadic MSI-H colon cancer were more likely to harbour synchronous sessile serrated adenomas ( 20 vs $8 \% ; P=0.023)$. This is the largest study to rigorously evaluate the precursor and synchronous lesions in patients with right-sided colon cancer. Detailed molecular and histological analysis of these lesions confirms the importance of serrated precursors in the development of sporadic MSI-H colon cancer.

Modern Pathology (2012) 25, 1423-1431; doi:10.1038/modpathol.2012.98; published online 8 June 2012
\end{abstract}

Keywords: BRAF; colorectal cancer; MSI-H; precursor; serrated

In the past 10 years, there has been a revolution in our understanding of colorectal cancer (CRC) owing to the identification of multiple distinct molecular pathways. Classically, CRC has been divided into cancers that exhibit chromosomal instability and those that exhibit microsatellite instability (MSI). ${ }^{1}$ More recently, CRCs that exhibit high levels of promoter methylation of $\mathrm{CpG}$ islands have been

Correspondence: RK Pai, MD, PhD, Department of Anatomic Pathology, Cleveland Clinic, 9500 Euclid Avenue, L25, Cleveland, OH 44195, USA.

E-mail: pair@ccf.org

Received 9 February 2012; revised 17 April 2012; accepted 18 April 2012; published online 8 June 2012 identified. CpG islands are present in the promoters of numerous genes, and when these islands are methylated, gene transcription is silenced. In $15 \%$ of sporadic CRCs, there is extensive methylation of $\mathrm{CpG}$ islands in the promoters of a wide variety of genes. ${ }^{2-4}$ For this reason, these tumors have been called CpG island methylator phenotype-high (CIMP-H) tumors. Approximately $60-80 \%$ of CIMP-H tumors also harbour mutations in BRAF, a serine threonine kinase in the RAS/RAF/MAPK pathway. ${ }^{5-9}$ These particular CRCs are also often characterized by high levels of microsatellite instability (MSI-H) ${ }^{5,8,10-16}$ owing to the presence of a CpG island in the promoter region of the mismatch repair protein gene $M L H 1$. 
Tubular, tubulovillous and villous adenomas (Ads) give rise to the majority of CRC, many of which exhibit chromosomal instability. Patients with Lynch syndrome also develop CRC through adenomas. However, it is believed that sporadic CIMP-H, MSI-H tumors arise through a unique serrated polyp termed sessile serrated adenomas (aka sessile serrated polyps; SSAs). For this reason, the pathway giving rise to CIMP-H, MSI-H tumors has been termed the serrated neoplasia pathway. SSAs share many molecular features with these tumors as they commonly harbour BRAF mutations (up to $90 \%$ ) and are CIMP-H.,.$^{5,8,11,12}$ By contrast, BRAF mutations have are extremely rare in conventional Ads. ${ }^{5,8,10-16}$ Thus, a mutation in BRAF serves as a specific marker for the serrated neoplasia pathway.

SSAs clearly do give rise to a proportion of sporadic MSI-H CRC as one occasionally finds an SSA immediately adjacent to the invasive carcinomas or preceding the CRC at the same site. Furthermore, the presence of SSAs increases the risk of advanced neoplasia at subsequent colonoscopies. ${ }^{17-19}$ The exact proportion of sporadic MSI-H CRCs that arise from an SSA, however, is unclear, and it remains possible that some of these tumors arise through another precursor lesion.

We recently described a group of atypical Ads with overlapping features with traditional serrated adenomas (TSAs) and SSAs that tend to occur in colons harbouring other serrated polyps. ${ }^{10}$ These atypical Ads exhibited focal serration and prominent eosinophilic cytoplasm. None of these atypical Ads harboured a BRAF mutation; however, they were more likely to have hypermethylation of CIMP-associated genes. It is possible that some sporadic MSI-H tumors arise through this unique polyp. Although these atypical Ads lack a BRAF mutation, they may acquire the mutation during the process of becoming invasive. Alternatively, it is possible that these atypical Ads give rise to those $20 \%$ of sporadic MSI-H CRCs that lack a BRAF mutation or those microsatellite stable (MSS) CRCs with low levels of CpG island methylation.

In an attempt to answer some of these questions, we reviewed 75 proximal MSI-H CRCs with loss of MLH1 expression, taking care to exclude those cases that are likely due to Lynch syndrome. For comparison, 89 right-sided MSS CRCs were also reviewed. The presence and type of adjacent precursor lesion was determined as was the type and number of synchronous polyps at the time of resection. BRAF mutational analysis was performed in all tumors, and the presence of a BRAF mutation was also determined in the adjacent precursor lesions. From these analyses, we attempted to determine what proportion of these sporadic MSI-H carcinomas was derived from a serrated precursor.

\section{Materials and methods}

\section{Study Population and MSI-H Analysis}

Beginning in 2005, CRCs with MSI-H histology (poor differentiation, tumor infiltrating lymphocytes, mucinous histology, and/or Crohn's-like reaction) were subjected to PCR-based MSI analysis. In 2009, this practice was made universal to all CRCs regardless of pathological features. Briefly, DNA from all tumors and matched normal specimens was obtained from formalin-fixed paraffin-embedded tissue. For each case, the hematoxylin and eosinstained tumor and paired normal tissue sections were reviewed by a pathologist to select the optimal area (at least 50\% tumor cells) for tissue microdissection. DNA was extracted using the High Pure PCR Template Preparation Kit (Roche Applied Science, Indianapolis, IN) according to the manufacturer's instructions. In cases where matched normal tissue was unavailable, DNA from peripheral blood samples was isolated using proteinase $\mathrm{K}$ digestion and phenol/chloroform extraction. The MSI test was performed using a fluorescent PCRbased assay (MSI Analysis System, Promega, Madison, WI) by amplifying 7 loci, including 5 mononucleotide repeats (BAT25, BAT26, NR-21, NR-24, and MONO-27) and two pentanucleotide repeats (PentaC and PentaD). ${ }^{20}$ The fluorescently labeled amplified PCR products were analyzed by capillary gel electrophoresis on the ABI 3100 Genetic Analyzer (Applied Biosystems, Foster City, CA). Per the manufacturer's instructions, a tumor exhibiting two or more microsatellite unstable markers was classified as MSI-H, cases with one unstable marker were classified as MSI-low, and those without any unstable markers were classified as MSS.

A search of the pathology database from 2005 to 2010 revealed 107 MSI-H CRCs. No MSI-low cases were identified using this method during this time period. After MSI analysis, MSI-H tumors were subjected to immunohistochemical staining using anti-MLH1 (clone G168.15 diluted 1:10; Biocare, Concord, CA) and anti-MSH2 (clone FE11, diluted 1:100; Biocare, Concord, CA). The presence or absence of nuclear staining was evaluated in the lesional tissue. The adjacent non-neoplastic epithelium, stromal cells, and lymphocytes were used as an internal control and stains were interpreted only when internal control tissue demonstrated distinct nuclear positivity. Loss of MMR protein expression was defined by lack of nuclear staining in the lesional tissue.

Only MSI-H CRCs with loss of MLH1 were included in this study. Furthermore, those at or distal to the splenic flexure were excluded. The Cleveland Clinic has an active genetic counselor service with particular interest in hereditary CRC. Review of the genetic counselors' clinical note taking into account family history (using modified Amsterdam criteria ${ }^{21}$ as a guide) and the presence of 
other Lynch-associated cancers was performed. MLH1 mutational analysis was performed in some cases as clinically indicated. In addition, any case meeting the criteria for hyperplastic/serrated polyposis ( $>5$ hyperplastic polyps/SSAs proximal to the sigmoid colon of which 2 are $>10 \mathrm{~mm}$ or $>30$ hyperplastic polyps distributed throughout the colon) was also excluded from the study. These criteria resulted in exclusion of $32(30 \%) \mathrm{MSI}-\mathrm{H}$ cases. The resulting 75 cases formed the cohort of sporadic right-sided MSI-H colon cancers. For comparison, 89 consecutive right-sided MSS colon cancers were also reviewed for the years 2009-10.

\section{Histological Review}

Hematoxylin and eosin-stained slides from 75 MSI$\mathrm{H}$ sporadic proximal colon cancers and $89 \mathrm{MSS}$ right-sided colon cancers were reviewed by two gastrointestinal pathologists (RKP and/or DTP). In addition to evaluating for the presence and type of immediate/adjacent precursor lesion, the type and number of synchronous polyps at the time of resection was also determined. As luminal extension of invasive colonic adenocarcinoma into the adjacent non-neoplastic mucosa may mimic highgrade dysplasia, and therefore a precursor lesion, only precursor lesions that were either large or demonstrated a transition in histological grade (lower grade compared with the adjacent invasive cancer), were considered as adjacent precursors. The size of the precursor lesions ranges from 4 to $25 \mathrm{~mm}$. The precursor lesions were categorized as Ads (including tubular, tubulovillous, and villous adenomas), SSAs with or without cytologic dysplasia, or TSAs using established criteria. ${ }^{22}$ Briefly, SSAs were identified by the presence of serrations at the base of the crypt, basilar crypt dilatation, horizontal orientation of the crypts, and lack of a distinct subepithelial-thickened collagen layer. ${ }^{23}$ TSAs were identified by prominent eosinophilic cytoplasm, serrated architecture present throughout the polyp, and abundant ectopic crypts. $^{24,25}$ In addition for all Ads, the following histological parameters were documented and graded in a semi-quantitative manner as follows: cytoplasmic eosinophilia $(0=$ none, $1=$ focal striking eosinophilia, $2=$ extensive striking eosinophilia), serrated crypts $(0=$ none, $1=1-3$ consecutive crypts with serrations, $2=>3$ consecutive crypts with serrations), ectopic crypts $(0=$ none, $1=<10 \%$ crypts within the entire precursor, $2=10-50 \%$ crypts, $3=>50 \%$ crypts), and villous component $(<25 \%$ tubular adenoma, 25-75\% tubulovillous adenoma, and $>75 \%$ villous adenoma).

\section{BRAF Mutation Analysis}

After careful manual microdissection of the area of interest, BRAF mutation analysis at codon 600
(V600E) was performed by a real-time PCR based on an allelic discrimination method as previously described. ${ }^{12}$ Briefly, real-time PCR was performed using allele-specific primers designed to selectively amplify the wild-type (T1796) and mutant (A1796) BRAF alleles. The primer sequences were as follows: V, 5'-GTGATTTTGGTCTAGCTACTGT; E, 5'-CGCGG CCGGCCGCGGCGGTGATTTTGGTCTAGCTACCGA; and AS, 5'-TAGCCTCAATTCTTACCATCCAC. PCR amplification and melting curve analysis were performed on an ABI 7500 (Applied Biosystems, Foster City, CA). Genomic DNA was amplified in a $20-\mu \mathrm{l}$ volume containing $1 \mathrm{X}$ Platinum SYBR Green qPCR SuperMix-UDG (Invitrogen, Carlsbad, CA, USA), forward primer V (300 nM), forward primer E (900 nM) and reverse primer AS (300 nM). The cycling conditions were as follows: $50^{\circ} \mathrm{C}$ for $2 \mathrm{~min}, 95^{\circ} \mathrm{C}$ for $2 \mathrm{~min}, 40$ cycles of $95^{\circ} \mathrm{C}$ for $15 \mathrm{~s}$ and $60^{\circ} \mathrm{C}$ for $60 \mathrm{~s}$. After amplification, samples were subjected to a temperature ramp from 60 to $99^{\circ} \mathrm{C}$, rising $1^{\circ} \mathrm{C}$ each step. For wild-type samples, single peaks were observed at $80^{\circ} \mathrm{C}$, while samples containing mutant alleles produced single peaks at $85^{\circ} \mathrm{C}$.

\section{Statistical Analysis}

Age was compared between the groups using the Student's $t$-test. Categorical variables were compared by Chi-square or Fisher's exact test, as appropriate. All statistical tests were two-sided, with $P$-values $<0.05$ considered statistically significant. Statistical analyses were performed using SAS software, version 9.1 (SAS Institute, Cary, NC, USA).

\section{Results}

\section{Subject Demographics and Tumor Characteristics}

A total of 75 sporadic right-sided MSI-H and 89 MSS colon cancers formed the study cohort. Male gender was more common in MSS colon cancers than is sporadic MSI-H cancers (61 vs $37 \%, P=0.003$ ). The median age of patients with sporadic MSI-H cancers (74 years; range 51-95 years) was significantly higher compared with patients with MSS cancers (67 years; range 40-95 years; $P<0.001$ ). Both the groups showed similar pT and $\mathrm{pN}$ stage distribution, the details of which are summarized in Table 1.

\section{Precursor Histology and Synchronous Lesions in MSI-H and Right-Sided MSS Tumors}

Of the 75 sporadic MSI-H tumors, 19 (25\%) cases demonstrated precursor lesions, of which 15 were Ads and 4 were SSAs with or without partial cytologic dysplasia (Table 2, Figure 1). In the MSS cohort, precursor lesions were identified in 31 (35\%) cases, 30 of which were Ads, whereas 1 precursor SSA was identified. Although precursor 
Table 1 Clinical and pathological features of the right-sided MSI$\mathrm{H}$ and MSS CRC

\begin{tabular}{lccr}
\hline Feature & MSI-H (n=75) & MSS (n=89) & P-value \\
\hline Median age & 74 & 67 & $<0.001$ \\
\% Male & $28(37 \%)$ & $54(61 \%)$ & 0.003 \\
& & & \\
pT stage & & & \\
T1 & $4(5 \%)$ & $8(9 \%)$ & 0.89 \\
T2 & $10(13 \%)$ & $10(11 \%)$ & \\
T3 & $38(51 \%)$ & $46(52 \%)$ & \\
T4a & $21(28 \%)$ & $22(25 \%)$ & \\
T4b & $2(3 \%)$ & $3(3 \%)$ & \\
& & & \\
pN stage & & & \\
NX & $1(1 \%)$ & $0(0 \%)$ & 0.25 \\
NO & $48(64 \%)$ & $43(48 \%)$ & \\
N1a & $6(8 \%)$ & $7(8 \%)$ & \\
N1b & $9(12 \%)$ & $19(21 \%)$ & \\
N2a & $4(5 \%)$ & $9(10 \%)$ & \\
N2b & $7(9 \%)$ & $11(12 \%)$ & \\
Adjacent precursor & $19(25 \%)$ & $31(35 \%)$ & 0.19 \\
BRAF mut & $57(76 \%)$ & $10(11 \%)$ & $<0.001$ \\
& & & \\
\end{tabular}

MSI-H, microsatellite instability-high; MSS, microsatellite stable.

SSAs were more common in the MSI-H group, this difference was of borderline significance (21 vs 3\%; $P=0.06$, Table 2). No precursor lesion meeting criteria for TSA was identified in either cohort.

Precursor Ads were carefully analyzed for atypical features, including serrations, cytoplasmic eosinophilia, ectopic crypts, and villous morphology. Precursor Ads of MSI-H CRC were more likely to demonstrate serrated crypt architecture compared with the MSS group $(P<0.001$; Table 3$)$. However, there was no difference in the proportion of ectopic crypts, the degree of cytoplasmic eosinophilia, or the presence of villous morphology in the precursor Ads between the two groups (Table 3, Figure 2).

The presence and type of synchronous polyps in both sporadic MSI-H and MSS CRCs are summarized in Table 2. Briefly, synchronous SSAs were more commonly found in patients with sporadic MSI-H tumors compared with those with MSS tumors (20 vs $8 \% ; P=0.023$ ). By contrast, MSS tumors were commonly associated with synchronous Ads compared with MSI-H tumors (47 vs $32 \%$; $P=0.048$ ). Appendiceal lesions were documented only in the MSI-H category (2 low-grade mucinous neoplasms and 1 SSA). There was no difference in the number of synchronous CRCs between the two groups.

\section{BRAF Mutation Analysis and Correlation with Precursor Histology}

BRAF mutations were significantly associated with sporadic MSI-H CRC compared with MSS CRC (76 vs $11 \% ; P<0.001$, Table 1). BRAF mutational analysis was also performed on all 19 precursors of MSI-H CRCs and compared with the mutation status of the adjacent CRC. Of the 15 precursor lesions
Table 2 Precursor histology and synchronous lesions to rightsided MSI-H and MSS CRC

\begin{tabular}{lccc}
\hline & MSI-H & MSS & P-value \\
\hline Precursor histology & $n=19$ & $n=31$ & \\
SSA with or without cytologic & $4(21 \%)$ & $1(3 \%)$ & 0.06 \\
dysplasia & & & \\
Ad (TA or TVA) & $15(79 \%)$ & $30(97 \%)$ & \\
Synchronous polyps/lesions & $n=75$ & $n=89$ & \\
Patients with SSAs & $15(20 \%)$ & $7(8 \%)$ & 0.023 \\
Patients with Ad & $24(32 \%)$ & $42(47 \%)$ & 0.048 \\
Patients with appendiceal lesions & $3(4 \%)$ & $0(0 \%)$ & 0.09 \\
Patients with synchronous CRC & $6(8 \%)$ & $4(4 \%)$ & 0.52 \\
& & & \\
\hline
\end{tabular}

MSI-H, microsatellite instability-high; MSS, microsatellite stable; SSA, sessile serrated adenoma; Ad, adenoma; TA, tubular adenoma; TVA, tubulovillous adenoma; CRC, colorectal cancer.

adjacent to BRAF-mutated MSI-H CRC, 14 harboured a BRAF mutation (3/3 SSAs and 11/12 Ads). No BRAF mutation was seen in the 4 precursor lesions adjacent to BRAF WT MSI-H CRCs (1 SSA and 3 Ads) (Figure 3).

In the MSS category, there were 10 BRAF-mutated CRC. A precursor lesion was identified in 4 cases in the form of 3 Ads and 1 SSA; all 4 precursor lesions $(100 \%)$ demonstrated BRAF mutation (Figure 3). Of the 3 BRAF-mutated Ads in the MSS cohort, 2 demonstrated areas of serration and all had areas of cytoplasmic eosinophilia. BRAF mutational analysis was not performed on the Ad precursor lesion of BRAF WT MSS CRC. However, of the 27 Ad adjacent to BRAF WT MSS CRC, 8 (30\%) demonstrated areas of cytoplasmic eosinophilia and crypt serrations.

\section{Discussion}

The efforts of numerous molecular studies have significantly improved our understanding of the pathways involved in colorectal carcinogenesis. The chromosomal instability pathway, a result of mutations of tumor suppressor genes and oncogenes, forms the basis of the Vogelstein's adenoma-carcinoma model of carcinogenesis. ${ }^{26}$ The other two pathways include the MSI pathway and CpG island methylation pathway. Epigenetic alterations in the form of methylation of the promoter regions of certain genes appear to be a common link between the sporadic MSI-H cancers and CIMP-H tumors. ${ }^{27,28}$ Another relative newcomer to the arena of colorectal carcinogenesis is the serrated neoplasia pathway. In fact, a unique aspect about this pathway is that most of these tumors demonstrate a CIMP-H and MSI-H phenotype. ${ }^{29}$

BRAF mutations are thought be an early event in the serrated neoplasia and sporadic MSI-H CRC pathway. BRAF mutations are commonly seen in microvesicular hyperplastic polyps, SSAs with and without cytologic dysplasia, and some TSAs. ${ }^{8}$ Given this finding, it has been assumed that all BRAFmutated CRC arise from a SSA or TSA. ${ }^{30-32}$ However, 

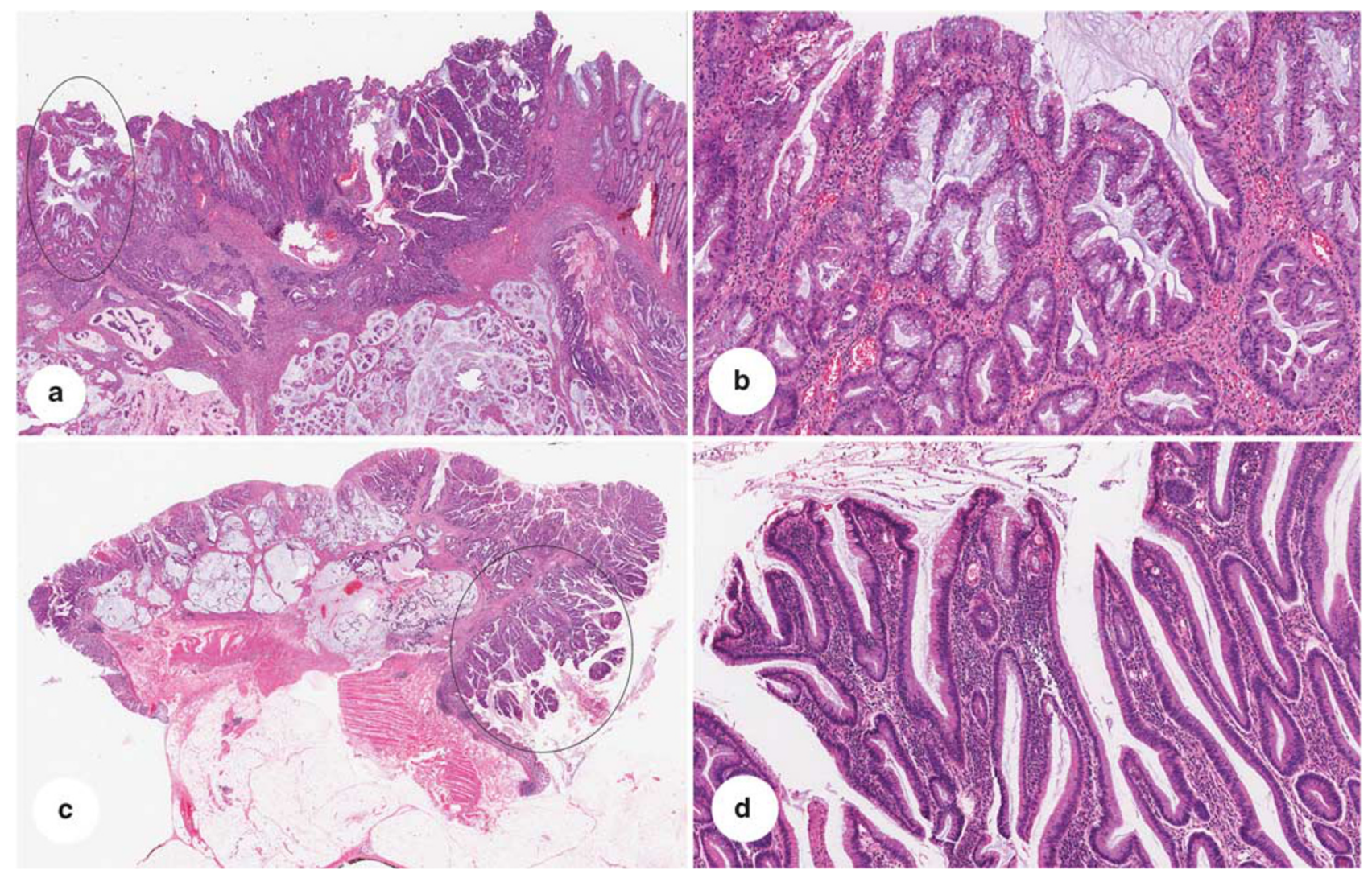

Figure 1 (a, b) Right-sided MSI-H invasive adenocarcinoma arising from an SSA with areas of cytologic dysplasia. Higher magnification of the precursor demonstrating serrated crypt architecture and dilated crypts (b). (c, d) An example of right-sided MSI-H invasive adenocarcinoma arising from an adenomatous precursor, in this case a tubulovillous adenoma (d) characterized by elongated, stratified, and hyperchromatic nuclei.

Table 3 Histological analysis of Ad precursors to right-sided MSI-H and MSS CRC

\begin{tabular}{lccc}
\hline Characteristic & $\begin{array}{c}\text { MSI-H } \\
\text { Precursors } \\
(\mathrm{n}=15)\end{array}$ & $\begin{array}{c}\text { MSS } \\
\text { precursors } \\
(\mathrm{n}=30)\end{array}$ & P-value \\
\hline $\begin{array}{l}\text { Eosinophilia } \\
0\end{array}$ & $6(40 \%)$ & $14(47 \%)$ & 0.92 \\
1 & $5(33 \%)$ & $8(27 \%)$ & \\
2 & $4(27 \%)$ & $8(27 \%)$ & \\
Serrations & & & \\
0 & $1(7 \%)$ & $19(63 \%)$ & $<0.001$ \\
1 & $5(33 \%)$ & $4(13 \%)$ & \\
2 & $9(60 \%)$ & $7(23 \%)$ & \\
Ectopic crypts & & & \\
0 & $6(40 \%)$ & $19(63 \%)$ & \\
1 & $4(27 \%)$ & $6(20 \%)$ & \\
2 & $3(20 \%)$ & $4(13 \%)$ & \\
3 & $2(13 \%)$ & $1(3 \%)$ & \\
Villous architecture & & & \\
TA & $2(13 \%)$ & $12(40 \%)$ & 0.09 \\
TVA & $13(87 \%)$ & $18(60 \%)$ & \\
\hline
\end{tabular}

MSI-H, microsatellite instability-high; MSS, microsatellite stable; TA, tubular adenoma; TVA, tubulovillous adenoma; CRC, colorectal cancer; WT, wild type. we have recently identified atypical Ads with a unique histology that occurs more commonly in patients with synchronous SSAs. ${ }^{10}$ These atypical Ads demonstrate focal serrations and cytoplasmic eosinophilia. None harboured a BRAF mutation, thus they do not represent SSAs that have been overgrown by dysplastic epithelium. However, these atypical Ads do demonstrate low levels of CIMP indicating that they may represent a novel precursor in the serrated neoplasia pathway. It is possible that some of these atypical Ads acquire a BRAF mutation late in their progression to CRC.

To determine what proportion of sporadic MSI-H tumors are derived from a serrated precursor vs atypical Ad, we compared 75 consecutive sporadic right-sided MSI-H colon cancers with 89 right-sided MSS cancers and systematically evaluated the tumors for the presence or absence of a precursor lesion (Ad, SSA, and TSA). All precursor Ads were assessed for focal serrations, cytoplasmic eosinophilia, and ectopic crypts. In addition, BRAF mutation status was performed on all invasive cancers and the corresponding precursor lesions in BRAF-mutated CRC and used as a surrogate for the serrated neoplasia pathway. We found that distinct precursors were recognized in $25 \%$ of sporadic MSI-H 

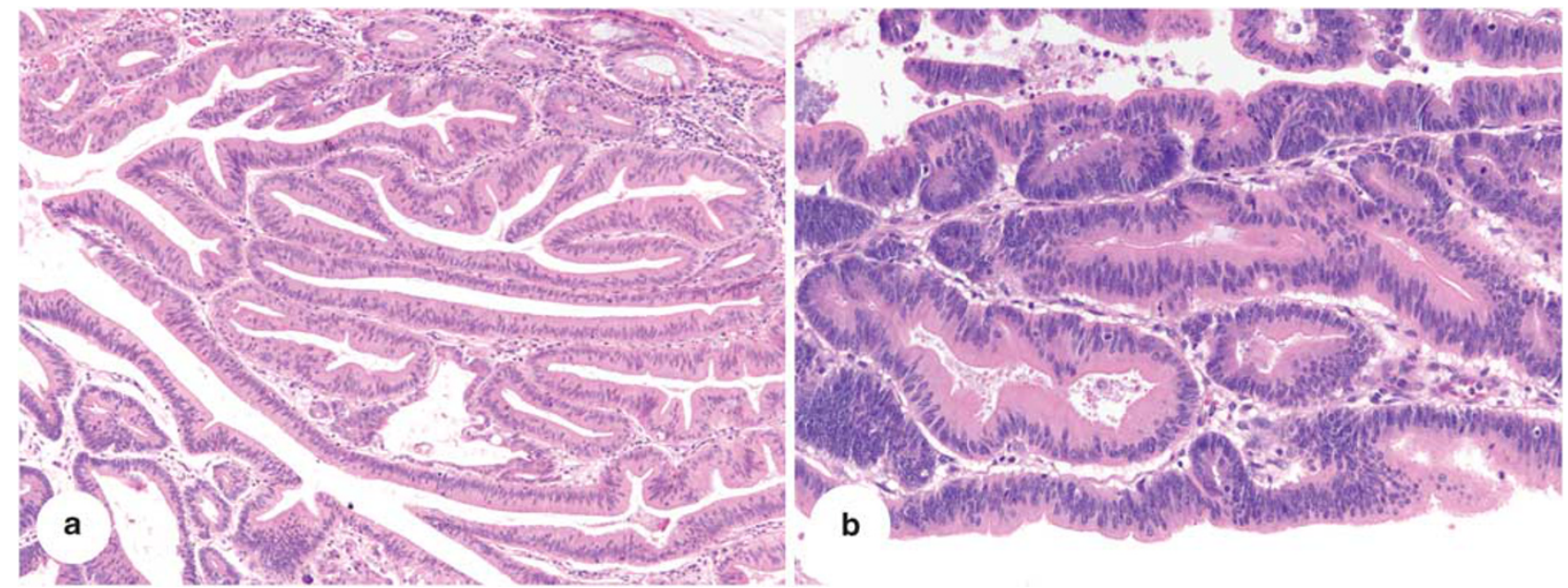

Figure 2 (a) Adenomatous precursor adjacent to an MSI-H cancer demonstrating cytoplasmic eosinophilia and serrated crypt architecture. (b) Another example of an adenomatous precursor showing ectopic crypts that do not extend to the muscularis mucosae.

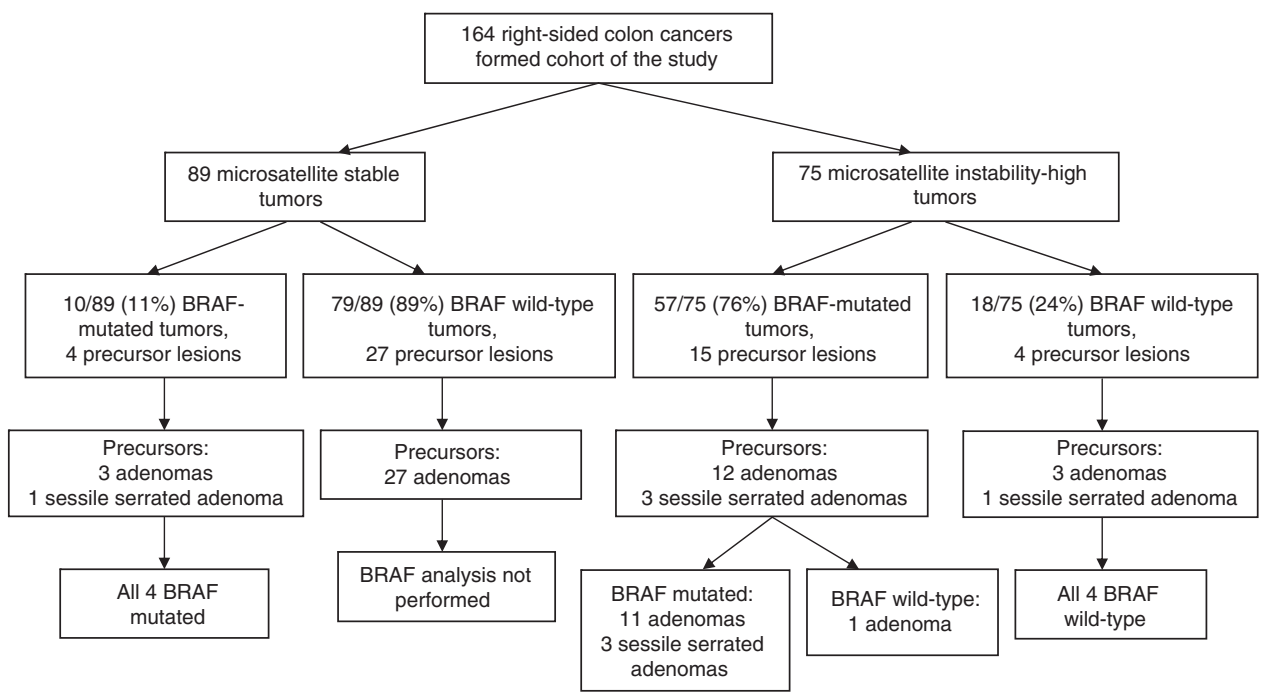

Figure 3 Flow chart detailing the precursor lesions and molecular analysis of right-sided MSI-H and MSS colon cancer.

cancers and $31 \%$ of MSS cancers. In the sporadic MSI-H cohort, SSAs were identified in 4/19 precursors $(21 \%)$ compared with $1 / 31(3 \%)$ in the MSS $(P=0.06)$. However, detailed histological analysis of the 15 precursor Ads to sporadic MSI-H CRC demonstrated epithelial serrations in 14 cases $(93 \%)$. This feature was significantly associated with MSI-H precursors compared with MSS precursors (Table 3). By contrast, cytoplasmic eosinophilia and ectopic crypts were not significantly different between these two cohorts.

It is possible that that the MSI-H Ad precursors with focal serrations identified in our study represent advanced SSAs that have become completely cytologically dysplastic. Alternatively, they may represent atypical Ads with focal serrations and cytoplasmic eosinophilia that we recently identified. It is possible that these atypical Ads acquire a
BRAF mutation as they become invasive. Review of 9 previous studies demonstrate that BRAF mutations are extremely rare in Ads (conventional and atypical) occurring in only 7 of 609 adenomas tested..$^{5,8,10-16}$ For this reason, BRAF mutation in a precursor lesion is sensitive and specific for SSAs. Of the 12 MSI-H Ad precursors associated with BRAF mutated CRC, 11 harboured a BRAF mutations. Similar results were seen in precursor Ads to BRAF-mutated MSS CRC in that all 3 precursor Ads were also BRAF mutated. These data strongly suggest that the overwhelming majority of BRAF-mutated MSI-H (at least 14/15; 93\%) and MSS $(4 / 4 ; 100 \%)$ CRC likely arise from an SSA, and by the time the carcinoma comes to clinical attention, the characteristic features of an SSA have been overgrown by cytologically dysplastic epithelium. Residual serrations remain in the 
dysplastic areas, possibly providing a clue to their origin from SSAs.

Two previous studies also attempted to determine the precursor lesion to MSI-H CRC. Young et al ${ }^{33}$ analyzed 47 sporadic MSI-H CRC, and a residual adenoma was seen in 13 cases of which 12 were classified as 'serrated adenomas'. BRAF mutational analysis was not done on these tumors or precursor lesion. The study by Young et al was performed before criteria for recognizing SSAs were proposed, and it is likely that the residual 'serrated adenomas' represent a mixture of SSAs and Ads with focal serrations (possible representing SSAs with complete cytologic dysplasia). In a more recent study, O'Brien et $a l^{8}$ analyzed 11 serrated carcinomas that were defined by the presence of residual 'serrated adenoma' as described by Longacre and FenoglioPressier $^{25}$ (now known as TSA). However, in 8 of these residual 'serrated adenomas', contiguous 'hyperplastic polyps' were seen. Of these 11 serrated carcinomas, 9 demonstrated microsatellite instability. O'Brien et al also analyzed BRAF mutations in the precursor and adjacent serrated carcinoma in 6 cases. All 6 serrated carcinomas and associated precursor lesions harboured BRAF mutations. In contrast to this previous study, we applied strict and current criteria for the diagnosis of SSA and TSA. Another major difference with O'Brien et al is that the current study analyzed 75 sporadic MSI-H CRC, where as O'Brien et al limited their study to 11 'serrated carcinomas' (of which only 9 were MSI-H). As serrated carcinomas were defined by the presence of a contiguous 'serrated adenoma' and not MSI-H, the study by O'Brien et al was not designed to determine the proportion of MSI-H CRC arise from a serrated precursor.

In the study by Young et al, molecular and histological analysis of MSI-H tumors was not stratified according to location within the colon. Similarly, O'Brien et al studied both proximal (4/11; $36.4 \%)$ and distal $(7 / 11 ; 63.6 \%)$ serrated carcinomas. The unique aspect of our study is that we specifically analyzed right-sided MSI-H and MSS tumors. This inclusion criterion was based on the fact that nearly $90 \%$ of sporadic MSI-H tumors occur in the proximal colon. ${ }^{33,34}$ Furthermore, there are differences in molecular alterations and serrated precursor lesions between the right and left colon. In particular, SSAs are uncommon in the left colon, whereas TSA are predominately left sided. ${ }^{23}$

In our cohort of 15 Ads adjacent to MSI-H tumors, 3 showed a wild-type BRAF status. All three lesions exhibited serrations in more than three crypts and one demonstrated extensive striking cytoplasmic eosinophilia. Although these BRAF WT MSI-H CRCs could have arisen through BRAFWT SSAs, the majority of SSAs harbour a BRAF mutation. Thus, it is possible that some of these CRCs arose through the atypical Ads that we recently described. ${ }^{10}$ Finally, a single BRAF-mutated CRC was associated with a BRAF-WT Ad precursor.
This result indicates that, in rare cases, BRAF mutations can be acquired relatively late in progression to CRC.

BRAF mutations have been documented in 5$20 \%$ of MSS cancers. ${ }^{2,29,35-37}$ In our study, BRAF mutation was documented in $11 \%$ of MSS cancers and although this data represents only right-sided MSS cancers, it is comparable to the reported literature, as in most studies this profile of tumors was predominantly encountered in the proximal colon. Like MSI-H tumors, BRAF mutations in MSS tumors have been correlated with CIMP-H phenotype. ${ }^{2,29,37}$ It was interesting to note that in the 10 MSS BRAF-mutated cancers, precursor lesions could be identified in 4 cases and all 4 precursors (1 SSA and 3 Ads) also harboured BRAF-mutation. Thus, BRAF-mutated MSS tumors also likely arise from a SSA that often becomes overgrown by dysplastic epithelium at the time of clinical presentation.

Interestingly, histological analysis of the precursor Ads to MSS BRAF-WT CRC demonstrated both crypt serrations and cytoplasmic eosinophilia in 8/ 27 (30\%) lesions. This percentage roughly correlates with the reported percentage of CIMP-low proximal CRC $(\sim 40 \%))^{29}$ It is possible that these lesions represent the atypical Ads that we recently identified with a CIMP-low phenotype; however, methylation analysis is necessary to support this hypothesis.

Lastly, compared with right-sided MSS tumors, the MSI-H tumors in this study were significantly associated with synchronous SSAs. Recent work has shown that patients with SSA are associated with a high percentage of synchronous serrated polyps (SSAs, SSAs with cytologic dysplasia, TSAs, and right-sided hyperplastic polyps). ${ }^{38}$ Although this study does not provide direct evidence in terms of molecular markers, the presence of synchronous lesions suggests that the entire colon in patients with sporadic MSI-H is predisposed to the serrated neoplasia pathway. Thus, the presence of synchronous SSAs in MSI-H CRC also indirectly supports the notion that SSAs give rise to these tumors.

In summary, in this retrospective comparative analysis of 75 proximal MSI-H and 89 MSS tumors, distinct precursors were identified in $25 \%$ of rightsided MSI-H tumors and $31 \%$ of MSS tumors. Although nearly one-fourth of these precursor lesions in MSI-H category were SSAs, the remainder lesions were composed entirely of cytologically dysplastic epithelium and best categorized as Ads. However, as these Ads also were mostly BRAF mutated, they likely represented advanced serrated precursors (SSAs with complete cytologic dysplasia). This is substantiated by the observation that serrated crypt architecture in the Ads precursors was the only histological feature that was significantly associated with MSI-H precursors compared with MSS precursors. To our knowledge, this is the largest study that analyzed the precursor lesions to right-sided CRC according to current histological 
criteria. The histological analysis coupled with molecular studies entirely confirms the importance of the serrated neoplasia pathway in the development of the majority of sporadic MSI-H CRC.

\section{Disclosure/conflict of interest}

The authors declare no conflict of interest.

\section{References}

1 Lengauer C, Kinzler KW, Vogelstein B. Genetic instability in colorectal cancers. Nature 1997;386:623-627.

2 Samowitz WS, Sweeney C, Herrick J, et al. Poor survival associated with the BRAF V600E mutation in microsatellite-stable colon cancers. Cancer Res 2005;65:6063-6069.

3 Herman JG, Umar A, Polyak K, et al. Incidence and functional consequences of hMLH1 promoter hypermethylation in colorectal carcinoma. Proc Natl Acad Sci USA 1998;95:6870-6875.

4 Kane MF, Loda M, Gaida GM, et al. Methylation of the hMLH1 promoter correlates with lack of expression of hMLH1 in sporadic colon tumors and mismatch repairdefective human tumor cell lines. Cancer Res 1997; 57:808-811.

5 Kambara T, Simms LA, Whitehall VL, et al. BRAF mutation is associated with DNA methylation in serrated polyps and cancers of the colorectum. Gut 2004;53:1137-1144.

6 Samowitz WS, Albertsen H, Herrick J, et al. Evaluation of a large, population-based sample supports a CpG island methylator phenotype in colon cancer. Gastroenterology 2005;129:837-845.

7 Weisenberger DJ, Siegmund KD, Campan M, et al. CpG island methylator phenotype underlies sporadic microsatellite instability and is tightly associated with BRAF mutation in colorectal cancer. Nat Genet 2006; 38:787-793.

8 O’Brien MJ, Yang S, Mack C, et al. Comparison of microsatellite instability, $\mathrm{CpG}$ island methylation phenotype, BRAF and KRAS status in serrated polyps and traditional adenomas indicates separate pathways to distinct colorectal carcinoma end points. Am J Surg Pathol 2006;30:1491-1501.

9 Shen L, Toyota M, Kondo Y, et al. Integrated genetic and epigenetic analysis identifies three different subclasses of colon cancer. Proc Natl Acad Sci USA 2007;104:18654-18659.

10 Pai RK, Mackinnon AC, Joseph L, et al. Identification of histologically distinct conventional adenomas that arise predominately in patients with sessile serrated adenomas. Am J Surg Pathol 2010;34:355-363.

11 Spring KJ, Zhao ZZ, Karamatic R, et al. High prevalence of sessile serrated adenomas with BRAF mutations: a prospective study of patients undergoing colonoscopy. Gastroenterology 2006;131:1400-1407.

12 Jass JR, Baker K, Zlobec I, et al. Advanced colorectal polyps with the molecular and morphological features of serrated polyps and adenomas: concept of a 'fusion' pathway to colorectal cancer. Histopathology 2006; 49:121-131.

13 Kakar S, Deng G, Cun L, et al. CpG island methylation is frequently present in tubulovillous and villous adenomas and correlates with size, site, and villous component. Hum Pathol 2008;39:30-36.

$14 \mathrm{Kim}$ K-M, Lee EJ, Ha S, et al. Molecular features of colorectal hyperplastic polyps and sessile serrated adenoma/polyps from Korea. Am J Surg Pathol 2011; 35:1274-1286.

15 Kim YH, Kakar S, Cun L, et al. Distinct CpG island methylation profiles and BRAF mutation status in serrated and adenomatous colorectal polyps. Int J Cancer 2008;123:2587-2593.

16 Carr NJ, Mahajan H, Tan KL, et al. Serrated and nonserrated polyps of the colorectum: their prevalence in an unselected case series and correlation of BRAF mutation analysis with the diagnosis of sessile serrated adenoma. J Clin Pathol 2009;62:516-518.

$17 \mathrm{Li} \mathrm{D}$, Jin C, McCulloch C, et al. Association of large serrated polyps with synchronous advanced colorectal neoplasia. Am J Gastroenterol 2009;104: 695-702.

18 Schreiner MA, Weiss DG, Lieberman DA. Proximal and large hyperplastic and nondysplastic serrated polyps detected by colonoscopy are associated with neoplasia. Gastroenterology 2010;139:1497-1502.

19 Lu F-I, van Niekerk de W, Owen D, et al. Longitudinal outcome study of sessile serrated adenomas of the colorectum: an increased risk for subsequent rightsided colorectal carcinoma. Am J Surg Pathol 2010; 34:927-934.

20 Bacher JW, Flanagan LA, Smalley RL, et al. Development of a fluorescent multiplex assay for detection of MSI-High tumors. Dis Markers 2004;20:237-250.

21 Vasen HF, Watson P, Mecklin JP, et al. New clinical criteria for hereditary nonpolyposis colorectal cancer (HNPCC, Lynch syndrome) proposed by the International Collaborative group on HNPCC. Gastroenterology 1999;116:1453-1456.

22 Snover DC, Ahnen DJ, Burt RW, et al. Serrated polyps of the colon and rectum and serrated polyposis. In: WHO Classification of Tumors of the Digestive System. 4th edn. World Health Organization Classification of Tumors. Lyon, France: International Agency for Research on Cancer; 2010, pp 160-165.

23 Torlakovic E, Skovlund E, Snover DC, et al. Morphologic reappraisal of serrated colorectal polyps. Am J Surg Pathol 2003;27:65-81.

24 Torlakovic EE, Gomez JD, Driman DK, et al. Sessile serrated adenoma (SSA) vs traditional serrated adenoma (TSA). Am J Surg Pathol 2008;32:21-29.

25 Longacre TA, Fenoglio-Preiser CM. Mixed hyperplastic adenomatous polyps/serrated adenomas. A distinct form of colorectal neoplasia. Am J Surg Pathol 1990; 14:524-537.

26 Vogelstein B, Fearon ER, Hamilton SR, et al. Genetic alterations during colorectal-tumor development. N Engl J Med 1988;319:525-532.

27 Jass JR, Whitehall VL, Young J, et al. Emerging concepts in colorectal neoplasia. Gastroenterology 2002;123:862-876.

28 Park S-J, Rashid A, Lee JH, et al. Frequent CpG island methylation in serrated adenomas of the colorectum. Am J Pathol 2003;162:815-822.

29 Ogino S, Nosho K, Kirkner GJ, et al. CpG island methylator phenotype, microsatellite instability, BRAF mutation and clinical outcome in colon cancer. Gut 2009;58:90-96.

30 Snover DC, Jass JR, Fenoglio-Preiser C, et al. Serrated polyps of the large intestine: a morphologic and 
molecular review of an evolving concept. Am J Clin Pathol 2005;124:380-391.

31 Jass JR. Classification of colorectal cancer based on correlation of clinical, morphological and molecular features. Histopathology 2007;50:113-130.

32 O’Brien MJ. Hyperplastic and serrated polyps of the colorectum. Gastroenterol Clin North Am 2007;36: 947-968, viii.

33 Young J, Simms LA, Biden KG, et al. Features of colorectal cancers with high-level microsatellite instability occurring in familial and sporadic settings: parallel pathways of tumorigenesis. Am J Pathol 2001;159:2107-2116.

$34 \mathrm{Kim} \mathrm{H}$, Jen J, Vogelstein B, et al. Clinical and pathological characteristics of sporadic colorectal carcinomas with DNA replication errors in microsatellite sequences. Am J Pathol 1994;145:148-156.
35 Rajagopalan H, Bardelli A, Lengauer C, et al. Tumorigenesis: RAF/RAS oncogenes and mismatch-repair status. Nature 2002;418:934.

36 Kakar S, Deng G, Sahai V, et al. Clinicopathologic characteristics, CpG island methylator phenotype, and BRAF mutations in microsatellite-stable colorectal cancers without chromosomal instability. Arch Pathol Lab Med 2008;132:958-964.

$37 \mathrm{Li} \mathrm{WQ}$, Kawakami K, Ruszkiewicz A, et al. BRAF mutations are associated with distinctive clinical, pathological and molecular features of colorectal cancer independently of microsatellite instability status. Mol Cancer 2006;5:2.

38 Pai RK, Hart J, Noffsinger AE. Sessile serrated adenomas strongly predispose to synchronous serrated polyps in non-syndromic patients. Histopathology 2010;56:581-588. 